\title{
Advantages of Autonomic Computing over Cloud Computing Comparative Analysis
}

\author{
${ }^{1}$ Samah Mawia Ibrahim Omer, ${ }^{2}$ Amin Babiker A.Mustafa, \\ ${ }^{3}$ Fatema Abdallah Elmahdi Alghali

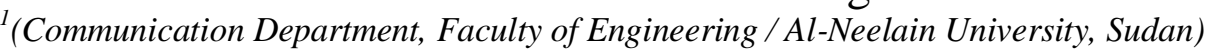 \\ Email: samahmawaya@gmail.com ) \\ ${ }_{2}^{2}$ Communication Department, Faculty of Engineering Dean, Al-Neelain University, Sudan) \\ Email: amin31766@gmail.com) \\ ${ }^{3}$ (Computer Department, Faculty of Science, Sebha University, Libya) \\ Email: fatmaaelgali@yahoo.com)
}

\begin{abstract}
The overarching goal of autonomic computing is to realize computer and software systems and applications that can manage themselves in accordance with high-level guidance from humans. Meeting the grand challenges of autonomic computing requires scientific and technological advances in a wide variety of fields, as well as new software and system architectures that support the effective integration of the constituent technologies. This paper presents an introduction to autonomic computing,
\end{abstract}

Its properties over cloud computing.

Keywords: cloud computing, autonomic computing, QoS.

\section{Introduction}

In these days there were time shared computing Systems such as cloud computing that: "refers to both the applications delivered as services over the Internet, and the hardware and system software in the data centers that provide those services", Cloud computing delivers infrastructure, platform, and software (applications) as subscription-based services in a pay-as-you-go model. In industry, these services are referred to as Infrastructure as a Service (IaaS), Platform as a Service (PaaS), and Software as Service (SaaS), respectively. Cloud infrastructure offering reliable, secure, and cost-efficient services is a Challenging task. It requires cooptimization at multiple layers (infrastructure, platform, and application) exhibiting autonomic properties. Challenges are:

- Quality of Service (QoS). Cloud service providers (CSPs) need to ensure that sufficient amount of resources are provisioned to ensure that QoS requirements of Cloud service consumers (CSCs) such as deadline, response time, and budget constraints are met.

-Energy efficiency. It includes having efficient usage of energy in the infrastructure, avoiding utilization of more resources than actually required by the application,

- Security. Achieving security features such as confidentiality (protecting data from unauthorized Access), availability (avoid malicious users making the application unavailable to legitimate users), and reliability against Denial of Service (DoS) attacks. The DoS is critical because, in a dynamic resource provisioning scenario, increase in the number of users causes automatic increase in the resources allocated to the application.

As Clouds are complex, large-scale, and heterogeneous distributed systems (e.g., consisting of multiple Data Centers, each containing 1000s of servers and peta-bytes of storage capacity), management is a crucial feature, which needs to be automated and integrated with intelligent strategies for dynamic provisioning of resources in an autonomic manner. Effective management of services becomes fundamental in platforms that constitute the fabric of computing Clouds; and to serve this purpose, autonomic models for PaaS (Platform As a Service) software systems are essential. Autonomic systems exhibit the ability of self-monitoring, Self-repairing and self-optimizing by constantly sensing themselves and tuning their performance. Such autonomic features are also exhibited by market economy, where resources/services are priced so as to maintain equilibrium in the supply and demand. Clouds constitute an interesting Venue to explore the use of autonomic features, because of their dynamism, large scale, and complexity.

\section{Cloud computing}

The main idea behind cloud computing is to make applications available on flexible execution Environments primarily located in the Internet. Cloud computing is of particular benefit to small and medium sized businesses who wish to completely outsource their data-center infrastructure, or large companies who wish 
to get peak load capacity without incurring the higher cost of building larger data centers internally. Cloud computing is an extension of this paradigm wherein the capabilities of business Applications are exposed as sophisticated services that can be accessed over a network. Cloud service providers are incentivized by the profits to be made by charging consumers for accessing these services. A Cloud is a type of parallel and distributed system consisting of a collection of inter-connected and virtualized computers that are dynamically provisioned and presented as one or more unified computing resources based on service - level agreements established through negotiation between the service provider and consumers ${ }^{[1]}$. Basically a cloud is of two types: Public Cloud, Private Cloud A public cloud sells services to anyone on the Internet. (The information and applications running on Public cloud may be accessed from the internet).A private cloud is a proprietary network or a data center That supplies hosted services to a limited number of people (the information and applications running on the private cloud may be accessed from the intranet via a VPN connection between the internal datacenter and the "outsourced" infrastructure).

The services ${ }^{[2]}$ provided by the cloud providers are:-

- SaaS - Software as a Service Network-hosted Application (By Google Apps, Salesforce.com)

- DaaS - Data as a Service Customer queries against provider's database (By Google Big Table, Amazon simpleDB)

- PaaS- Platform as a Service Network - hosted software development platform (By Windows Azure, Google App Engine)

- IaaS - Infrastructure as a Service Provider hosts customer VMs or provides network Storage (By Amazon web service EC2, Go grid, Rack space)

-IPMaaS - Identity and Policy Management as a Service Provider manages identity and/or Access control policy for customer (By Right scale, Appistry)

-NaaS - Network as a Service

- Provider offers virtualized networks (e.g. VPNs)

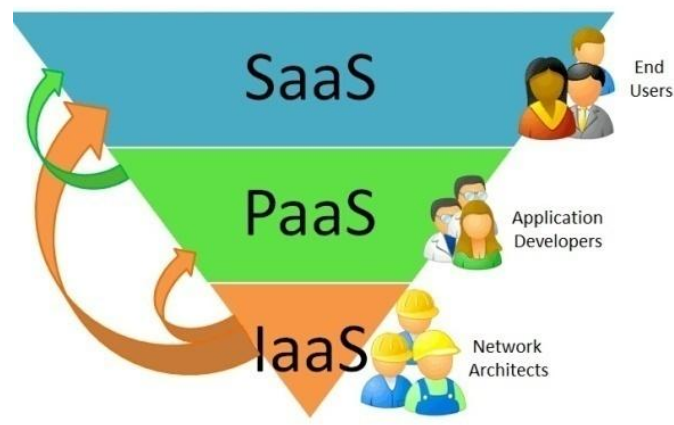

Figure (1) cloud computing ${ }^{[5]}$

III. Autonomic computing

The only option remaining is autonomic computing - computing systems that can manage themselves given high-level objectives from administrators. When IBM's senior vice president of research, Paul Horn, introduced this idea to the National Academy of Engineers at Harvard University in a March 2001 keynote address, he deliberately chose a term with a biological connotation. The autonomic nervous system governs our heart rate and body temperature, thus freeing our conscious brain from the burden of dealing with these and many other low-level, yet vital, functions The term autonomic computing is emblematic of a vast and somewhat tangled hierarchy of natural self-governing systems, many of which consist of myriad interacting, self-governing components that in turn comprise large numbers of interacting, autonomous, self-governing components at the next level down. The enormous range in scale, starting with molecular machines within cells and extending to human markets, societies, and the entire world socioeconomic, mirrors that of computing systems, which run from individual devices to the entire Internet. Thus, we believe it will be profitable to seek inspiration in the self-governance of social and economic systems as well as purely biological ones. Clearly then, autonomic computing is a grand challenge that reaches far beyond a single organization. Its realization will take a concerted, long-term, worldwide effort by researchers in a diversity of fields. A necessary first step is to examine this vision: what autonomic computing systems might look like, how they might function, and what obstacles researchers will face in designing them and understanding their behavior. 


\subsection{Self-Management}

The essence of autonomic computing systems is self-management, the intent of which is to free system administrators from the details of system operation and maintenance and to provide users with a machine that runs at peak performance 24/7. Like their biological namesakes, autonomic systems will maintain and adjust their operation in the face of changing components, workloads, demands, and external conditions and in the face of hardware or software failures, both innocent and malicious. The autonomic system might continually monitor its own use, and check for component upgrades, for example. If it deems the advertised features of the upgrades worthwhile, the system will install them, reconfigure itself as necessary, and run a regression test to make sure all is well. When it detects errors, the system will revert to the older version while its automatic problem determination algorithms try to isolate the source of the error. Figure 1 illustrates how this process might work for an autonomic accounting system upgrade. IBM frequently cites four aspects of self-management; early autonomic systems may treat these aspects as distinct, with different product teams creating solutions that address each one separately. Ultimately, these aspects will be emergent properties of a general architecture, and distinctions will blur into a more general notion of self-maintenance. The journey toward fully autonomic computing will take many years, but there are several important and valuable milestones along the path. At first, automated functions will merely collect and aggregate information to support decisions by human administrators. Later, they will serve as advisors, suggesting possible courses of action for humans to consider. As automation technologies improve, and our faith in them grows, we will entrust autonomic systems with making - and acting on-lower-level decisions. Over time, humans will need to make relatively less frequent predominantly higher-level decisions, which the system will carry out automatically via more numerous, lower level decisions and actions. Ultimately, system administrators and end users will take the benefits of autonomic computing for granted. Self-managing systems and devices will seem completely natural and unremarkable, as will automate software and middleware upgrades. The detailed migration patterns of applications or data will be as uninteresting to us as the details of routing a phone call through the telephone network.

\subsection{Self-configuration}

Installing, configuring, and integrating large, complex systems is challenging, time-consuming, and error-prone even for experts. Most large Web sites and corporate data centers are haphazard accretions of servers, routers, databases, and other technologies on different platforms from different vendors. It can take teams of expert programmers' months to merge two systems or to install a major e-commerce application such as SAP. Autonomic systems will configure themselves automatically in accordance with high-level policies representing business-level objectives, for example - that specify what is desired, not how it is to be accomplished. When a component is introduced, it will incorporate itself seamlessly, and the rest of the system will adapt to its presence - much like a new cell in the body or a new person in a population. For example, when a new component is introduced into an autonomic accounting system, as in Figure 1, it will automatically learn about and take into account the composition and configuration of the system. It will register itself and its capabilities so that other components can either use it or modify their own behavior appropriately.

\subsection{Self-optimization}

Complex middleware, such as Web Sphere, or database systems, such as Oracle or DB2, may have hundreds of tunable parameters that must be set correctly for the system to perform optimally, yet few people know how to tune them. Such systems are often integrated with other, equally complex systems. Consequently, performance-tuning one large subsystem can have unanticipated effects on the entire system. Autonomic systems will continually seek ways to improve their operation, identifying and seizing opportunities to make themselves more efficient in performance or cost. Just as muscles become stronger through exercise, and the brain modifies its circuitry during learning, autonomic systems will monitor, experiment with, and tune their own parameters and will learn to make appropriate choices about keeping functions or outsourcing them. They will proactively seek to upgrade their function by finding, verifying, and applying the latest updates.

\subsection{Self-healing}

IBM and other IT vendors have large departments devoted to identifying, tracing, and determining the root cause of failures in complex computing systems. Serious customer problems can take teams of programmers several weeks to diagnose and fix, and sometimes the problem disappears mysteriously without any satisfactory diagnosis. Autonomic computing systems will detect, diagnose, and repair localized problems resulting from bugs or failures in software and hardware, perhaps through a regression tester, as in Figure 1. Using knowledge about the system configuration, a problem- diagnosis component (based on a Bayesian network, for example) would analyze information from $\log$ files, possibly supplemented with data from 
additional monitors that it has requested. The system would then match the diagnosis against known software Patches (or alert a human programmer if there are none), install the appropriate patch, and retest.

\subsection{Self-protection}

Despite the existence of firewalls and intrusion detection tools, humans must at present decide how to protect systems from malicious attacks and inadvertent cascading failures. Autonomic systems will be selfprotecting in two senses. They will defend the system as a whole against large-scale, correlated problems arising from malicious attacks or cascading failures that remain uncorrected by self-healing measures. They also will anticipate problems based on early reports from sensors and take steps to avoid or mitigate them. ${ }^{[3]}$

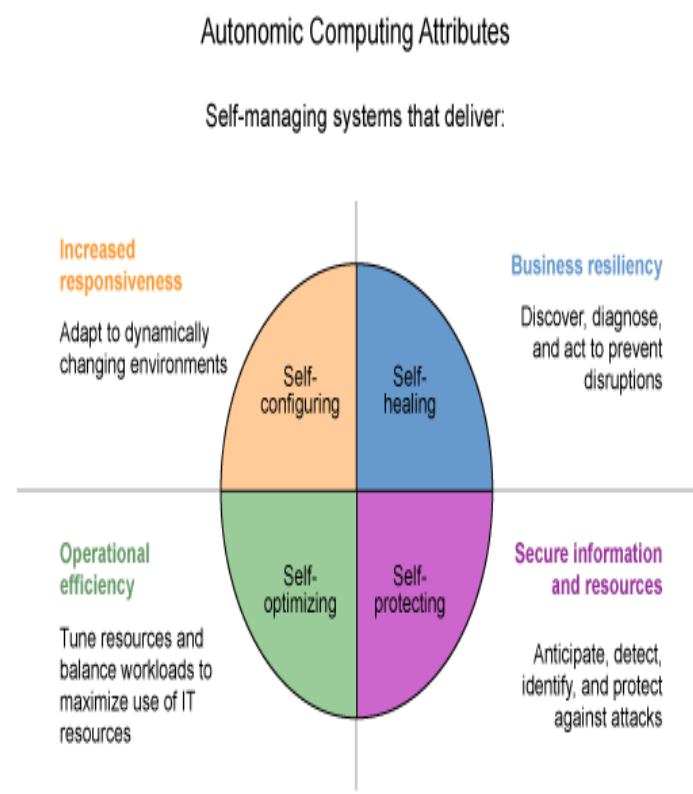

Figure (2) autonomic computing [6]

Differentiating Cloud Computing And Auotnomic Computing ${ }^{[4]}$

\begin{tabular}{|c|c|c|}
\hline Comparative view & Cloud computing & Autonomic computing \\
\hline Characteristics & $\begin{array}{l}\text { 1\Dynamic computing infrastructure } \\
2 \backslash \text { Minimally or self managed platform } \\
3 \backslash \text { Consumption based billing }\end{array}$ & $\begin{array}{l}\text { 1\Self-Configuration } \\
2 \backslash \text { Self-Healing } \\
\text { 3\Self-Optimization } \\
4 \backslash \text { Self-Protection }\end{array}$ \\
\hline Location & $\begin{array}{l}\text { Computers do not have to be in the same } \\
\text { physical location }\end{array}$ & $\begin{array}{l}\text { Computers do not have to be in the same } \\
\text { physical location }\end{array}$ \\
\hline Operating system & $\begin{array}{l}\text { The memory, storage devices and net work } \\
\text { communications are managed by the OS of the } \\
\text { basic physical cloud units }\end{array}$ & $\begin{array}{l}\text { All Computers controlled by individual } \\
\text { OS Composed Error Correction And } \\
\text { Detection }\end{array}$ \\
\hline Node indecency & Every node acts as an independent entity & Every node acts as an independent entity \\
\hline Net work & Clouds are mainly distributed over MAN & $\begin{array}{l}\text { Autonomics are mainly distributed over } \\
\text { WAN }\end{array}$ \\
\hline processing system & $\begin{array}{l}\text { Allows Multiple Smaller Applications To Run } \\
\text { At the same Times }\end{array}$ & 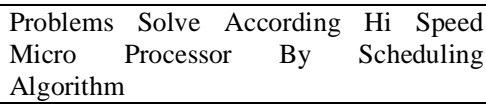 \\
\hline Computing area & $\begin{array}{l}\text { 1/ Banking } \\
\text { 2/ Insurance } \\
\text { 3/ Weather for Casting } \\
\text { 4/ Space Exploration } \\
\text { 5/ SAAS } \\
\text { 6/ PAAS } \\
\text { 7/ IAAS }\end{array}$ & $\begin{array}{l}\text { 1/ Composite resources tied to business } \\
\text { decision-making } \\
\text { 2/ Composite resources decision-making, } \\
\text { e.g., cluster servers } \\
\text { 3/ Resource elements managing } \\
\text { themselves }\end{array}$ \\
\hline
\end{tabular}

\section{Discussion}

The table contains many comparatives views discuss the improvement in the autonomic computing VS cloud computing in characteristics, operating system, node independency. Processing system and computing area.

Two Computing are agree in the computers do not have to be in the same physical location 


\section{Conclusion}

The future for autonomic computing is bright. The big companies in computers are throwing lots of resources into this. Company like Amazon DELL, HP and Intel etc sees a huge market for autonomic computing in the future. One of the biggest benefits of autonomic, of course, is costing less money than buying the application outright. The service provider can offer cheaper, more reliable applications / resources than organizations can buy themselves automatically.

\section{References:}

[1]. Future Generation Computer Systems25(2009)599-616 www.elsevier.com/locate/fgcs. The University of Melbourne, Australia

[2]. http://www.dolcera.com/wiki/index.php?title=Cloud_Computing\#Cloud_computing_comparison_of_different_vendors

[3]. autonomic computing self protection the vision of autonomic computing [1 1 Jeffrey O,kephart 2ldavid M.chess]

[4]. cloud computing over cluster., grid computing comparative analysis-indugandotra,pawanesh Abrol,poja Gupta Rohit Uppal and sandeep Singh-Iournal of grid and distributed computing-volumee 1Issue1,2011

[5]. www.cloud computing.com/image

[6]. Www.autonomic computing.com/image

[7]. classical.istrbuted,cluster,grid, utility and cloud computing[1\Amin babiker ALNabi 2\Osama Abdallah Mmohammed Enan 3 lyassebMuhammed Tom Yusif] 